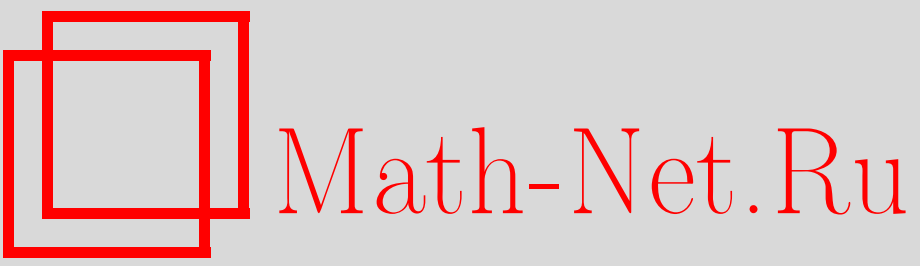

В. В. Козлов, Т. В. Мадсен, Равномерное распределение и сходимость по Вороному, Матем. сб., 2005, том 196, номер 10, 103-110

DOI: https://doi.org/10.4213/sm1427

Использование Общероссийского математического портала Math-Net.Ru подразумевает, что вы прочитали и согласны с пользовательским соглашением

http://www.mathnet.ru/rus/agreement

Параметры загрузки:

IP : 34.239 .49 .27

26 апреля 2023 г., 17:20:06 


\section{Равномерное распределение и сходимость по Вороному}

Имеется широкое обобщение равномерно распределенной последовательности по Вейлю, когда частота попадания элементов этой последовательности в интервал определяется с использованием матричного метода суммирования общего вида. В работе указаны условия равномерного распределения в случае, когда в качестве метода суммирования берется регулярний метод Вороного. Доказательства основаны на оценках тригонометрических сумм некоторого специального вида. Показано, что последовательность дробных долей логарифмов натуральных чисел не является равномерно распределенной ни при каком выборе регулярного метода Вороного.

Библиографоял: 6 названий.

\section{§1. Средние Вороного и типы равномерного распределения}

Пусть $q_{0}>0, q_{n} \geqslant 0$. Для последовательности $s_{n}, n=0,1, \ldots$, положим

$$
u_{n}=\frac{q_{n} s_{0}+q_{n-1} s_{1}+\cdots+q_{0} s_{n}}{q_{0}+q_{1}+\cdots+q_{n}} .
$$

Ясно, что если $s_{j}=s_{0}$, то $u_{n}=s_{0}$. Последовательность $q_{n}$ задает метод суммирования Вороного: если $u_{n} \rightarrow s$ при $n \rightarrow \infty$, то по определению

$$
s_{n} \rightarrow s \quad\left(W, q_{n}\right) .
$$

Критерий регулярности $W$-метода:

$$
\frac{q_{n}}{q_{0}+\cdots+q_{n}} \rightarrow 0
$$

Оказывается, всякие два регулярных метода Вороного совместимы, т.е. если $s_{n} \rightarrow s(W)$ и $s_{n} \rightarrow s^{\prime}\left(W^{\prime}\right)$, то $s=s^{\prime}$.

При $q_{0}=q_{1}=\cdots$ метод Вороного переходит в метод Чезаро. Если $W$-метод регулярен, последовательность $q_{n}$ не убывает, то $\left(W, q_{n}\right)$ включает метод Чезаро $C$ : из $s_{n} \rightarrow s(C)$ вытекает, что $s_{n} \rightarrow s\left(W, q_{n}\right)$. Больший интерес представляют условия обратного включения. Пусть $q_{0}=1, q_{n} \geqslant 0$. Если $W$-метод регулярен, $q_{n}$ не возрастает и

$$
\frac{q_{n+1}}{q_{n}} \geqslant \frac{q_{n}}{q_{n-1}}
$$

для всех $n \geqslant 1$, то $C$ включает $\left(W, q_{n}\right)$.

Работа выполнена в рамках Программы поддержки ведущих научных школ РФ (грант № 
Теория суммирования Вороного подробно изложена в [1].

Пусть $x_{n}, n=0,1,2, \ldots,-$ последовательность точек единичного отрезка $[0,1]$, $L \subset[0,1]$ - произвольный отрезок длины $l, f$ - характеристическая функция этого отрезка.

Возьмем некоторый метод суммирования Вороного $\left(W, q_{n}\right)$. Будем говорить, что последовательность точек $x_{n}\left(W, q_{n}\right)$-равномерно распределена по mod1 (кратко: $\left(W, q_{n}\right)$-рp или $\left.W-p p\right)$, если для каждого отрезка $L$

$$
f\left(x_{n}\right) \rightarrow l \quad\left(W, q_{n}\right) .
$$

Это определение отличается от классического определения Вейля тем, что каждое событие $x_{n} \in L$ учитывается со своим весом. При $q_{0}=q_{1}=\cdots$ получим определение последовательности, равномерно распределенной по Вейлю. Можно дать более обшее определение равномерного распределения, заменяя метод Вороного некоторьм линейньм методом суммирования (см., например, [2]).

Легко доказывается, что соотношение (1.2) справедливо для всех интегрируемых по Риману функций $f$, только $l$ надо, конечно, заменить интегралом от $f$ по отрезку $0 \leqslant x \leqslant 1$. Как и в классическом случае, справедливо обращение этого результата: если для всех целых $m \neq 0$

$$
e^{2 \pi i m x_{n}} \rightarrow 0 \quad\left(W, q_{n}\right),
$$

то последовательность $x_{n}$ будет $\left(W, q_{n}\right)$-pp.

Ясно, что всякая последовательность, равномерно распределенная в обобщенном смысле, всюду плотна на отрезке $[0,1]$. Обратное, конечно, не верно. Например, как будет показано в $\S 4$, последовательность $\{\alpha \ln n\}, \alpha \neq 0$, всюду плотна, но, вообще говоря, не является $W$-pp ни для какого регулярного $W$-метода. Здесь и далее $\{x\}$ обозначает дробную часть числа $x$. Отметим еше, что условие регулярности (1.1) необходимо для сушествования хотя бы одной $W$-рp последовательности [3]. По-видимому, это условие также является достаточным.

\section{§ 2. Условия $W$-равномерного распределения}

Теорема 1. Пусть непрерввно дифференцируемъе функции $f(x), g(x), x \geqslant 0$, удовлетворяют следующим условиям:

1)

$$
f^{\prime} \neq 0 \quad u \quad f^{\prime}(x) \rightarrow 0 \quad \text { npu } \quad x \rightarrow \infty ;
$$

2) положительная функция $g$ монотонна и

$$
\begin{gathered}
\int_{0}^{\infty} g(x) d x=\infty, \\
\frac{g(x)}{\int_{0}^{x} g(t) d t} \rightarrow 0 \quad n p u \quad x \rightarrow \infty ;
\end{gathered}
$$

3) отношение $g(n-x) / f^{\prime}(x)$ не убъвает или не возрастает на отрезке $0 \leqslant x \leqslant n$ 
4)

$$
\left|f^{\prime}(x)\right| \int_{0}^{x} g(t) d t \rightarrow \infty \quad \text { npu } \quad x \rightarrow \infty .
$$

Тогда последовательность $\{f(n)\}-(W, g(n))-p p$.

Условие (2.2) является условием регулярности метода Вороного $(W, g(n))$. Из этой теоремы можно вывести ряд следствий.

СлЕДСтвиЕ 1. В предположениях теоремы 1 последовательность $\{f(n)\}$ всюду плотна на единичном отрезке.

СлЕДСтвИЕ 2 (теорема Фейера [4]). Если $f^{\prime}(x)$ монотонно стремится $\kappa$ нулю и $x\left|f^{\prime}(x)\right| \rightarrow \infty$ при $x \rightarrow \infty$, то последовательность $\{f(n)\}$ равномерно распределена по Вейлю.

Для доказательства надо положить $g(x) \equiv 1$.

СлЕДСТВИЕ 3. Пусть $f^{\prime}>0, f^{\prime}(x) \rightarrow 0$ монотонно, $g(x)>0$ не возрастает $u$

$$
f^{\prime}(x) \int_{0}^{x} g(t) d t \rightarrow \infty
$$

при $x \rightarrow \infty$. Тогда $\{f(n)\}-(W, g(n))-p p$.

Действительно, условие 1) теоремы 1 , очевидно, вьполнено. Далее, интеграл (2.1) расходится, иначе нарушается условие $(2.3)$ (поскольку $f^{\prime} \rightarrow 0$ ). Условие (2.2) также выполнено ввиду ограниченности $g$ и уже установленного свойства (2.1). Далее, отношение $g(n-x) / f^{\prime}(x)$ не убывает в интервале $[0, n]$ как произведение двух неубывающих функций. Наконец, условие 4) теоремы 1 совпадает c (2.3).

Положим, например, $g(x)=(x+1)^{-\alpha}, 0 \leqslant \alpha<1$. Тогда условие (2.3) примет вид

$$
x^{1-\alpha} f^{\prime}(x) \rightarrow \infty .
$$

Если $f^{\prime}(x)>0$ и монотонно стремится к нулю, то это условие гарантирует, что последовательность $\{f(n)\}$ будет $\left(W,(n+1)^{-\alpha}\right)$-рp. Легко проверить, что $\left(W,(n+1)^{-\alpha}\right)$-методы при всех $0 \leqslant \alpha<1$ включены в метод Чезаро. В частности, последовательность $\{f(n)\}$ будет равномерно распределенной по Вейлю. При $\alpha \rightarrow 0$ условие (2.4) переходит в условие Фейера (следствие 2 ). Положим теперь $\alpha=1$. Тогда (2.4) заменяется условием

$$
(\ln (x+1)) f^{\prime}(x) \rightarrow \infty .
$$

В этом случае последовательность $\{f(n)\}-(W, 1 /(n+1))-p p$.

Особый интерес представляет случай, когда функция $g$ возрастает. Тогда регулярньй $(W, g(n))$-метод включает метод Чезаро. Однако здесь, как правило, условие (1.3) теоремы 1 не выполняется.

Укажем одну из возможных модификаций теоремы 1. Будем считать далее, что функция $f$ дважды непрерывно дифференцируема. Положим $\varphi(x)=1 / f^{\prime}(x)$. Если 
продифференцировать произведение $\varphi(x) g(n-x)$ по $x$ и разделить результат на это произведение, то получим

$$
\frac{\varphi^{\prime}(x)}{\varphi(x)}-\frac{g^{\prime}(n-x)}{g(n-x)} .
$$

Предположим, что функции $\varphi^{\prime} / \varphi$ и $g^{\prime} / g$ монотонно убьвают до нуля при $x \rightarrow \infty$. В частности, $f^{\prime \prime} / f^{\prime} \rightarrow 0$ монотонно. Тогда при достаточно больших $n$ в интервале $[0, n]$ функция (2.5) имеет ровно один нуль, который мы обозначим $x_{n}$.

ТЕОРема 2. Пусть выполнены следующие условия:

1) $f>0, f^{\prime}(x) \rightarrow 0, f^{\prime \prime}(x) / f^{\prime}(x)$ монотонно стремится $\kappa$ нулю при $x \rightarrow \infty$

2) $g>0, g$ монотонно возрастает, а $g^{\prime}(x) / g(x) \rightarrow 0$ монотонно при $x \rightarrow \infty$

3)

$$
\frac{g(n)}{f^{\prime}\left(x_{n}\right) \int_{0}^{n} g(t) d t} \rightarrow 0, \quad \frac{g\left(x_{n}\right)}{f^{\prime}(n) \int_{0}^{n} g(t) d t} \rightarrow 0
$$

nрu $n \rightarrow \infty$.

Тогда последовательность $\{f(n)\}$ будет $(W, g(n))$-рр.

СлЕДСТВИЕ 4. Пусть выполнены условия 1), 2) теоремы 2 и

$$
\frac{g(x)}{f^{\prime}(x) \int_{0}^{x} g(t) d t} \rightarrow 0
$$

при $x \rightarrow \infty$. Тогда $\{f(n)\}-(W, g(n))-p p$.

Действительно, с учетом предположений 1) и 2) из (2.7) следует условие (2.6).

СлЕДСТВИЕ 5. Пусть $f^{\prime}>0$, функиии $f^{\prime}(x)$ u $f^{\prime \prime}(x) / f^{\prime}(x)$ монотонно стремятся $\kappa$ нулю при $x \rightarrow \infty u$

$$
f^{\prime}\left(\frac{x}{2}\right) f^{\prime}(x) \int_{0}^{x} \frac{d t}{f^{\prime}(t)} \rightarrow \infty .
$$

Тогда $\{f(n)\}-\left(W, 1 / f^{\prime}(n)\right)-p p$.

Легко проверить, что в этом случае все условия теоремы 2 выполнены, причем $x_{n}=n / 2$. При этом условия (2.6) переходят в (2.8).

Условие (2.8) можно заменить более сильным:

$$
\left(f^{\prime}(x)\right)^{2} \int_{0}^{x} \frac{d t}{f^{\prime}(t)} \rightarrow \infty
$$

По правилу Лопиталя оно сводится к условию

$$
\frac{f^{\prime \prime}(x)}{\left(f^{\prime}(x)\right)^{2}} \rightarrow 0
$$

ПРИМЕР. Положим $f(x)=\ln ^{\alpha}(x+1), \alpha>0$. Условие (2.9) вьполняется лишь при $\alpha>1$. В этом случае последовательность $\left\{\ln ^{\alpha}(n+1)\right\}$ будет $W-p p$ при подходяшем выборе регулярного $W$-метода. Однако, как будет показано в $\S 4$, это свойство теряется при $\alpha=1$. Таким образом, огрубленное достаточное условие $W-p p$ на самом деле близко к необходимому. 


\section{§3. Доказательства}

Докажем сначала теорему 1. Согласно (1.3) нам надо показать, что каждая из сумм

$$
\begin{aligned}
& g(n) \sin 2 \pi f(0)+\cdots+g(0) \sin 2 \pi f(n), \\
& g(n) \cos 2 \pi f(0)+\cdots+g(0) \cos 2 \pi f(n)
\end{aligned}
$$

есть

$$
o\left(\sum_{s=0}^{n} g(s)\right)
$$

Для определенности оценим первую сумму (3.1), вторая сумма оценивается аналогично. Положим $g(x)=G(n-x)$ или, что то же самое, $G(x)=g(n-x)$. Для удобства $2 \pi f$ будем обозначать кратко $f$.

Сначала покажем, что (3.2) можно заменить на

$$
o\left(\int_{0}^{n} g(x) d x\right)
$$

Для этого воспользуемся формулой суммирования Эйлера-Маклорена:

$$
g(0)+\cdots+g(n)=\int_{0}^{n} g(x) d x+\frac{1}{2}(g(0)+g(n))+O\left(\int_{0}^{n}\left|g^{\prime}(x)\right| d x\right)
$$

По предположению функция $g$ монотонна. Следовательно,

$$
\int_{0}^{n}\left|g^{\prime}\right| d x=\left|\int_{0}^{n} g^{\prime} d x\right|=|g(n)-g(0)|
$$

Разделим обе части равенства (3.3) на интеграл от функции $g$ :

$$
\frac{\sum_{0}^{n} g(s)}{\int_{0}^{n} g d x}=1+O\left(\frac{g(0)}{\int_{0}^{n} g d x}\right)+O\left(\frac{g(n)}{\int_{0}^{n} g d x}\right) .
$$

Каждое из отношений, стоящих под знаком $O$, стремится к нулю, когда $n \rightarrow \infty$ : первое - по условию (2.1), а второе - по условию (2.2). Следовательно, при $n \rightarrow \infty$ левая часть (3.4) стремится к единице. Откуда вытекает требуемое.

Для оценки первой суммы (3.1) снова воспользуемся формулой Эйлера-Маклорена:

$$
\begin{aligned}
& \sum_{s=0}^{n} G(s) \sin f(s)=\int_{0}^{n} G(x) \sin f(x) d x \\
& \quad+\frac{1}{2}(G(0) \sin f(0)+G(n) \sin f(n))+O\left(\int_{0}^{n}\left|(G(x) \sin f(x))^{\prime}\right| d x\right) .
\end{aligned}
$$


Оценим сначала интеграл в правой части (3.5). Так как $f^{\prime} \neq 0$ (условие 1$)$ ), то можно сделать замену переменной $f(x)=t$. Тогда

$$
\int_{0}^{n} G(x) \sin f(x) d x=\int_{f(0)}^{f(n)} \frac{G}{f^{\prime}} \sin t d t .
$$

Согласно условию 3$)$ отношение $G / f^{\prime}$ монотонно зависит от новой переменной $t$. По второй теореме о среднем интеграл (3.6) равен

$$
\left.\frac{G}{f^{\prime}}\right|_{f(0)} \int_{f(0)}^{\xi} \sin t d t+\left.\frac{G}{f^{\prime}}\right|_{f(n)} \int_{\xi}^{f(n)} \sin t d t,
$$

причем $f(0) \leqslant \xi \leqslant f(n)$. Ясно, что

$$
\left.\frac{G}{f^{\prime}}\right|_{f(0)}=\left.\frac{G\left(f^{-1}(t)\right)}{f^{\prime}\left(f^{-1}(t)\right)}\right|_{t=f(0)}=\frac{G(0)}{f^{\prime}(0)} .
$$

Аналогично,

$$
\left.\frac{G}{f^{\prime}}\right|_{f(n)}=\frac{G(n)}{f^{\prime}(n)} .
$$

Так как интеграл от синуса по абсолютной величине не превосходит 2, то интеграл (3.6) оценивается величиной

$$
2 \frac{G(0)}{\left|f^{\prime}(0)\right|}+2 \frac{G(n)}{\left|f^{\prime}(n)\right|}=2 \frac{g(n)}{\left|f^{\prime}(0)\right|}+2 \frac{g(0)}{\left|f^{\prime}(n)\right|}
$$

При делении на интеграл

$$
\int_{0}^{n} g(x) d x
$$

оба этих слагаемых стремятся к нулю, когда $n \rightarrow \infty$. Первое - в силу условия регулярности (2.2), а второе - в силу условия 4).

По тем же причинам

$$
\frac{G(0) \sin f(0)+G(n) \sin f(n)}{\int_{0}^{n} g d x} \rightarrow 0
$$

при $n \rightarrow \infty$

Наконец, оценим

$$
\int_{0}^{n}\left|(G \sin f)^{\prime}\right| d x \leqslant \int_{0}^{n}\left|G^{\prime}\right| d x+\int_{0}^{n} G\left|f^{\prime}\right| d x
$$

Так как $G$ монотонно, то

$$
\int_{0}^{n}\left|G^{\prime}\right| d x=|g(n)-g(0)| .
$$


Следовательно,

$$
\frac{\int_{0}^{n}\left|G^{\prime}\right| d x}{\int_{0}^{n} g d x} \rightarrow 0 .
$$

Поскольку $\left|f^{\prime}(x)\right| \rightarrow 0$ при $x \rightarrow \infty$ (условие 1$)$ ), то по правилу Лопиталя

$$
\frac{\int_{0}^{n} G\left|f^{\prime}\right| d x}{\int_{0}^{n} G d x} \rightarrow 0 \text {. }
$$

Таким образом, теорема 1 полностью доказана.

Теорема 2 доказывается точно так же, как и теорема 1. Только теперь интервал $[0, n]$ разбивается на два интервала $\left[0, x_{n}\right]$ и $\left[x_{n}, n\right]$, в каждом из которых отношение $g(n-x) / f^{\prime}(x)$ монотонно. Применяя в каждом из этих интервалов теорему о среднем, получаем следуюшую оценку интеграла (3.7):

$$
2 \frac{G\left(x_{n}\right)}{\left|f^{\prime}(0)\right|}+2 \frac{G(0)}{\left|f^{\prime}\left(x_{n}\right)\right|}+2 \frac{G(n)}{\left|f^{\prime}\left(x_{n}\right)\right|}+2 \frac{G\left(x_{n}\right)}{\left|f^{\prime}(n)\right|} .
$$

В силу условий теоремы 2 после деления на интеграл (3.8) каждое из этих слагаемых стремится к нулю при $n \rightarrow \infty$.

\section{§4. О последовательности дробных долей логарифмов}

ТеОРема 3. Пусть $a \geqslant 2$ целое $и W$ - регулярный метод суммирования Вороного. Тогда последовательность

$$
\left\{\frac{1}{2} \log _{a} n\right\}
$$

не является $W-p p$.

ЗАмЕЧАНИЕ. По-видимому, этот результат справедлив для любой последовательности $\{\alpha \ln n\}, \alpha \neq 0$.

ДОКАЗАТЕЛЬСТво ТЕОРемЫ 3. Рассмотрим последовательность $s_{n}, n \geqslant 1$, которая определяется по следуюшему правилу: $s_{n}=1$, если

$$
a^{2 m} \leqslant n<a^{2 m+1},
$$

$m \geqslant 0$ целое; $s_{n}=0$ в остальных случаях. Покажем, что если последовательность (4.1) $W-p p$, то

$$
s_{n} \rightarrow \frac{1}{2}(W) .
$$

Действительно, логарифмируя неравенство (4.2), получаем

$$
m \leqslant \frac{1}{2} \log _{a} n<m+\frac{1}{2},
$$

откуда

$$
0 \leqslant\left\{\frac{1}{2} \log _{a} n\right\}<\frac{1}{2}
$$


Следовательно, $s_{n}=1$ тогда и только тогда, когда $\left\{\left(\log _{a} n\right) / 2\right\}$ принадлежит интервалу $L=[0,1 / 2)$ длины $l=1 / 2$. Применяя $(1.2)$, получаем требуемое.

Покажем теперь, что последовательность $s_{n}$ вообще не является $W$-сходяшейся. Ее элементы - частичные суммы ряда

$$
u_{1}+u_{2}+\cdots
$$

где $u_{a^{2 m}}=1, u_{a^{2 m+1}}=-1$, а остальные члены равны нулю. Поставим в соответствие числовому ряду (4.4) степенной ряд

$$
\sum u_{k} x^{k}=x-x^{a}+x^{a^{2}}-x^{a^{3}}+\cdots .
$$

Воспользуемся одним общим результатом, восходящим к Вороному (см. [1; теорема 18]). Если $W$-метод регулярен и выполнено (4.3), то определяемая рядом (4.5) функция стремится к $1 / 2$, ког да $x \rightarrow 1$ по вешественным значениям, меньшим чем 1 . Однако хорошо известно (см., например, [1; п. 4.10]), что при $a>1$ функция (4.5) вообще не стремится ни к какому пределу, когда $x \rightarrow 1$.

ЗАмЕчАниЕ. Как показано в [5], последовательность дробных долей логарифмов $(R, 1 /(n+1))$ - $p p$. Здесь $\left(R, p_{n}\right)$ - метод суммирования Рисса: $s_{n} \rightarrow s\left(R, p_{n}\right)$, если

$$
\frac{p_{0} s_{0}+p_{1} s_{1}+\cdots+p_{n} s_{n}}{p_{0}+p_{1}+\cdots+p_{n}} \rightarrow s .
$$

Теорема 3 приводит к предположению, что $(R, 1 /(n+1))$-метод включает все регулярные методы Вороного.

В [5] указаны достаточные условия обшего характера на дифференцируемые функции $f$ и $g$, при которых последовательность $\{f(n)\}$ оказывается $(R, g(n))$ - $p p$. В работе [6] приведены несколько иные условия. Теорема 1 является аналогом результатов [5], [6] для метода Вороного.

\section{Список литературы}

1. Харди Г. Расходящиеся ряды. М.: ИЛ, 1951.

2. Кейперс Л., Нидеррейтер Г. Равномерное распределение последовательностей. М.: Наука, 1985.

3. Горделий E.K. K теореме фон Неймана о перестановках всюду плотных последовательностей // Вестн. МГУ. Сер. 1. Матем., мех. 2004. №6. С. 18-24.

4. Гашков С.Б., Чубариков В.Н. Арифметика. Алгоритмы. Сложность выгислений. М.: Наука, 1996.

5. Tsuji M. On the uniform distribution of numbers $\bmod 1 / /$ J. Math. Soc. Japan. 1952. V. 4. P. 313-322.

6. Козлов В. В. О равномерном распределении // Изв. вузов. Северо-Кавказский регион. Сер. естеств. науки. 2001. С. 96-99. 